\title{
NEW TECHNIQUES FOR THE STUDY OF STOCHASTIC EQUILIBRIUM PROCESSES
}

\author{
Lawrence E. BLUME* \\ University of Michigan, Ann Arbor, MI 48109, USA
}

Received February 1979, final version accepted December 1980

\begin{abstract}
This paper develops the notion of transition correspondences; the set-valued analog of transition probabilities. A generalization of the Feller property for transition probabilities is shown to imply the existence of a selection from the transition correspondence having a stationary equilibrium. These techniques are applied to the existence problem for Markov temporary equilibrium processes in place of assumptions about the existence of continuous selections from the equilibrium price correspondence.
\end{abstract}

\section{Introduction}

In this paper I propose a method of constructing Markov processes of temporary equilibrium states that provides a useful alternative to the usual approach [Grandmont and Hildenbrand (1974) and Blume (1977)]. In this new method I construct transition correspondences, the set-valued analog of transition probabilities. Properties such as the existence of stationary equilibria are studied by using the hemi-continuity properties of the transition correspondence rather than continuity or stability properties of some pre-chosen selection from the equilibrium correspondence. It will be seen that the required upper hemi-continuity of the transition correspondence is often more easily established than the existence of a selection from the equilibrium correspondence having the desired properties.

The usual approach to this question involves constructing a correspondence defining equilibrium states. A selection is chosen from this correspondence and used to construct a transition probability. Desirable properties for the transition probability such as the Feller property can be inferred from properties of the selection, and these properties in turn imply the existence of an invariant probability measure for the transition probability. The technique proposed in this paper avoids all difficulties concerning the existence of well-behaved selections by working directly with the equilibrium correspondence. Theorems $2.1,3.1$ and 3.2 taken together in

\footnotetext{
*The author is grateful for comments received from the referee, and especially grateful to Jim Jordan for finding an error in an earlier version of this paper.
} 
effect prove the existence of a selection from the equilibrium correspondence such that the transition probability constructed from it has an invariant probability measure.

In section 2, Markov transition correspondences are defined, and sufficient conditions for the existence of an invariant measure are given. Section 3 shows how to construct Markov transition correspondences satisfying the sufficient conditions of section 2. An application of the results to stochastic equilibrium is offered in section 4 .

\section{Invariant probability measures}

In this section I show that convex-valued upper hemi-continuous transition correspondences have fixed points. The dependence of fixed points on parameters is also studied. Let $X$ and $Z$ be subsets of complete, separable metric spaces, with $X$ compact and $Z$ closed. Let $C(X)$ denote the set of all continuous functions from $X$ into the real line, and let $D$ denote the unit ball in $C(X)$ (with the sup norm). Let $\sigma(X)$ denote the $\sigma$-field of Borel subsets of $X$, and let $M(X)$ denote the set of all probability measures on $(X, \sigma(X))$, topologized with the topology of weak convergence. Also, $\sigma(M(X))$ is the Borel $\sigma$-field on $M(X)$.

Transition probabilities are particular examples of stochastic kernels, and so I begin by defining multivalued stochastic kernels:

Definition 2.1. A multivalued stochastic kernel (m.s.k.) is a correspondence $K: Z \rightarrow M(X)$ with measurable graph.

This definition generalizes the stochastic kernel concept. A stochastic kernel is a function $\hat{K}: Z \times \sigma(X) \rightarrow \boldsymbol{R}$ such that $K(z, \cdot) \in M(X), \forall z \in Z$, and $K(\cdot, A)$ is measurable for all $A \in \sigma(X)$. If an m.s.k. is singleton valued, then the measurable graph condition implies that the function $K: Z \rightarrow M(X)$ is measurable. Define $\hat{K}: Z \times \sigma(K) \rightarrow R$ such that $\hat{K}(z, A)=K(z)(A)$. The maps $\lambda_{\mathrm{A}}: \mu \rightarrow \mu(A), \forall \mu \in M(X)$, are measurable for all $A \in \sigma(X)$, so $\hat{K}(\cdot, A)$ is measurable. Since $\hat{K}(z, \cdot)=K(z) \in M(X), \hat{K}$ is a stochastic kernel.

The action of an m.s.k. $K$ on a measurable function $f$ is defined by applying to $f$ measurable selections from $K$. If $g: Z \rightarrow M(X)$ is a measurable selection from $K$, I will write $g \sim K$.

Definition 2.2. If $f: X \rightarrow \boldsymbol{R}$ is measurable, then $\int f(x) K(z, \mathrm{~d} x)=\beta_{f}(z)$ $=\left\{\int f(x) g(z, \mathrm{~d} x) ; g \sim K\right\}$.

If $K$ were a stochastic kernel and $f$ a bounded measurable real-valued function, then $\beta_{f}(Z)$ would be a bounded measurable real-valued function. An analogous property holds for m.s.k.'s. 
Proposition 2.1. If $K$ is an m.s.k. and $f$ a bounded measurable real-valued function, then $\beta_{f}$ is a correspondence with $(\sigma(Z) \times \sigma(\boldsymbol{R}))$ analytic graph.

Proof. The map $\mu \rightarrow \int f \mathrm{~d} \mu$ is measurable. The conclusion follows from Hildenbrand (1974, D.II.3, proposition 2). Q.E.D.

The Feller property for stochastic kernels states that if $f \in C(X)$, then $\beta_{f} \in C(Z)$. This property is a key sufficient condition for the existence of invariant probability measures for transition probabilities. An analogous property for m.s.k.'s plays the same role in the study of multivalued transition probabilities:

Definition 2.3. An m.s.k. $K$ has the multivalued Feller property if for all $f \in C(X), \beta_{f}: Z \rightarrow R$ is upper hemi-continuous (u.h.c.).

The Feller property for stochastic kernels implies that the map $K: Z \rightarrow M(X)$ is continuous. This result generalizes.

Proposition 2.2. An m.s.k. $K$ has the multivalued Feller property, iff $K$ is upper hemi-continuous.

Proof. Let $I$ denote the interval $[-1,1]$. The map $\lambda: M(X) \rightarrow \Pi_{f \in D} I$ defined such that $\lambda(\mu)_{f}=\int f \mathrm{~d} \mu$ is continuous with respect to the product topology and is a homeomorphism of $M(X)$ onto $\lambda(M(X))$. Thus it suffices to show that $\lambda \cdot K: Z \rightarrow \Pi_{f \in D} I$ is u.h.c.

It suffices to show that $\{z \in Z: \lambda \cdot K(z) \subset A\}$ is open for all $A$ in some subbase of $\Pi_{f \in D} I$. Consider the sub-base of sets of the form $A_{f}^{\prime}=\Pi_{f \in D} B(f)$ where $B_{f}=I$ if $f \neq f^{\prime}$, and $B_{f}^{\prime}$ is open in $I$. Then $\left\{z: \lambda \cdot K(z) \subset A_{f}^{\prime}\right\}=\left\{z: \beta_{f}(z)\right.$ $\left.\subset B_{f}^{\prime}\right\}$ which is open since $\beta_{f}$ is u.h.c.

Conversely, if $K$ is u.h.c., then $\beta_{f}$ has closed graph. Since $f$ is bounded, range $\beta_{f}$ is compact and so $\beta_{f}$ is u.h.c. Q.E.D.

Stochastic kernels also act upon probability measures. If $K$ is a stochastic kernel, then $\int K(z, A) \mathrm{d} \mu=v(A)$ defines a probability measure $v$. A similar action can be defined for m.s.k.'s.

Definition 2.4. If $\mu \in M(X)$ then $\gamma(\mu)(A)=\int K(z)(A) \mathrm{d} \mu=\left\{\int g(z)(A) \mathrm{d} \mu\right.$; $g \sim K\}$.

Note that $\gamma(\mu) \subset M(X)$, and $\gamma(\mu) \neq \phi$ since, from the Aumann selection theorem, there exists an (a.e. $\mu$ ) selection $g$ from $K$.

The Feller property for stochastic kernels implies that $\gamma$ is a continuous function. This result also generalizes. 
Proposition 2.3. If an m.s.k. $K$ has the multivalued Feller property, then $\gamma: M(Z) \rightarrow M(X)$ is u.h.c.

Proof. The idea is the same as that in Proposition 2.2. It suffices to show that $\lambda \cdot \gamma$ is u.h.c. The set

$$
\begin{aligned}
\left\{\mu: \lambda \cdot \gamma(\mu) \subset A_{f}\right\} & =\left\{\mu: \int_{x} f(x) \int_{z} g(z, \mathrm{~d} x) \mathrm{d} \mu \subset B_{f}, \forall f \in D, g \sim K\right\} \\
& =\left\{\mu: \int_{x} f^{\prime}(x) \int_{z} g(z, \mathrm{~d} x) \mathrm{d} \mu \subset B_{f}^{\prime}, \forall g \sim K\right\} \\
& =\left\{\mu: \int_{z} \int_{x} f^{\prime}(x) g(z, \mathrm{~d} x) \mathrm{d} \mu \in B^{\prime f}, \forall g \sim K\right\} \\
& =\left\{\mu: \int_{z} \beta_{f}(z) \mathrm{d} \mu \subset B_{f}^{\prime}\right\} .
\end{aligned}
$$

Thus it suffices to show that for all $f \in D$, the correspondence $\mu \rightarrow \int \beta_{f}(z) \mathrm{d} \mu$ is u.h.c. Since $\beta_{f}: Z \rightarrow I$, I need only show that this correspondence has closed graph. Let $\mu_{n} \rightarrow \mu_{0}$, and applying Skohorod's theorem, there exists a probability space $(S, \Sigma, \nu)$ and measurable maps $h_{n}, h_{0}$ such that $\mu_{n}=\nu \cdot h_{n}^{-1}$, $\mu_{0}=\nu \cdot h_{0}$, and $h_{n} \rightarrow h$ a.e. $\nu$. From Hildenbrand (1974, D.II.4, theorem 5) conclude that $\int \beta_{f} \mathrm{~d} \mu_{n}=\int \beta_{f} \cdot h_{n} \mathrm{~d} \nu_{0}$ for $n \geqq 0$. Then his D.II.4, theorem 6, implies that $\int \beta_{f} \mathrm{~d} \mu$ has closed graph. Q.E.D.

So far, the definition of the domain of the stochastic kernel has been general. Now I want to explicitly consider multivalued transition probabilities. In applications I might want to study a parametric class of multivalued transition probabilities and see how the invariant measures depend on the parameter.

Definition 2.5. A parametric multivalued transition probability (p.m.t.p.) is a multivalued stochastic kernel $K: X \times Z \rightarrow M(X)$.

The main result of this paper is the following theorem:

Theorem 2.1. Let $K$ be a convex valued p.m.t.p. satisfying the multivalued Feller property. Then for all $\nu \in M(Z)$ there exists $\mu \in M(X)$ such that $\mu \in$ $\gamma(\mu \times \nu)$. Furthermore, the correspondence $\nu \rightarrow\{\mu: \mu \in \gamma(\mu \times \nu)\}$ is u.h.c.

Neglecting the parameters, this theorem says that, under the given hypotheses, there exists a selection $g \sim K$ and a $\mu \in M(X)$ such that for all $A \in \sigma(X), \mu(A)=\int g(x)(A) \mathrm{d} \mu$. A transition probability having an invariant probability can be selected from $K$. The selection $g$ need not satisfy the Feller property, and in fact no selection satisfying it may exist.

Proof. Fix $v$. The map $\mu \rightarrow \mu \times v$ is continuous, so from Proposition 2.3, the correspondence $\mu \rightarrow \gamma(\mu \times v)$ is non-empty and u.h.c. If $K$ is convex-valued, then so is $\gamma(\mu \times v)$. For let $\delta, \delta^{\prime} \in \gamma(\mu \times v)$. Then there exists $g, g^{\prime} \sim K$ such that $\delta(\cdot)=\int \mathrm{g}(x, z)(\cdot) \mathrm{d}(\mu \times v), \delta^{\prime}(\cdot)=\int g^{\prime}(x, z)(\cdot) \mathrm{d}(\mu \times v)$. Then $\alpha \delta+(1-\alpha) \delta^{\prime}(\cdot)$ $=\int \alpha g+(1-\alpha) g^{\prime}(\cdot) \mathrm{d}(\mu \times v)$, and $\alpha g+(1-\alpha) g^{\prime} \sim K . \quad M(X)$ is convex and compact, and so Ky Fan's fixed point theorem implies the existence of $\mu \epsilon$ $\gamma(\mu \times \nu)$. Since $\gamma$ is u.h.c. and $(\mu, \nu) \rightarrow \mu \times \nu$ is continuous, the fixed point correspondence is u.h.c. Q.E.D. 


\section{Identifying convex-valued p.m.t.p.'s with the multivalued Feller property}

Multivalued stochastic kernels amenable to the analysis of section 2 arise in a variety of economic settings. In this section I identify an important source of these kernels - those arising as distributions of a correspondence with respect to a transition probability. As before, let $X$ be compact and $Z$ closed. Furthermore, let $E$ be a compact subset of a complete separable metric space.

Assumption 3.1. Let $\phi: X \times Z \times E \rightarrow X$ be a non-empty valued u.h.c. correspondence.

Assumption 3.2. Let $\psi: X \rightarrow M(E)$ be a map with the following two properties:

(i) $\psi$ is continuous,

(ii) $\mu \in$ range $\psi$ implies that $\mu$ is atomless.

Definition 3.1. Let $K(x, z)=\psi(x) \cdot \phi^{-1}=\left\{\mu \in M(X): \mu=\psi(x) \cdot g^{-1}(\cdot, x, z)\right.$ for some $g \sim \phi\}$.

The main results of this section are:

Theorem 3.1. If $\phi$ satisfies Assumption 3.1 and $\psi$ satisfies Assumption 3.2.i, then $K$ has the multivalued Feller property.

Theorem 3.2. If $\phi$ satisfies Assumption 3.1 and $\psi$ satisfies Assumption 3.2.i, ii, then $K$ is convex valued.

Thus if Assumptions 3.1 and 3.2 are satisfied, the hypotheses of Theorem 2.1 are satisfied.

Proof of Theorem 3.1. Since $X$ is compact, it suffices to show that $K: X \times Z$ $\rightarrow M(X)$ has closed graph. So, let $\left\{\left(x_{n}, y_{n}\right)\right\}_{n=1}^{\infty}$ be a sequence with limit $\left(x_{0}, y_{0}\right)$. Skorohod's theorem says that there exists a probability space $(\Omega, a, \lambda)$ and measurable functions $f_{n}: \Omega \rightarrow E, n \geqq 0$ such that $f_{n} \rightarrow f_{0}$ d.e. $\lambda$ and $\psi\left(x_{n}\right)=\lambda \cdot f_{n}^{-1}$. Without loss of generality a negligible set can be removed from $\Omega$ so that convergence is sure in the topology of pointwise convergence on $E^{\Omega 2}$. Thus, let $F=\bigcup_{n=0}^{\infty}\left\{f_{n}\right\}$. Then $F \subset E^{\Omega}$ is compact. Let $Y$ $=\bigcup_{n=0}^{\infty}\left\{\left(x_{n}, z_{n}\right)\right\}$-and $Y(w)=\bigcup_{n=0}^{\infty}\left\{\left(x_{n}, z_{n}, f_{n}(w)\right)\right\}$. These sets are compact in $X \times Z$ and $X \times Z \times E$, respectively.

Let $G_{n}=\left\{g: E \rightarrow X, g \sim \phi\left(x_{n}, z_{n}, \cdot\right)\right\}$ for $n=0,1, \ldots$. Then since $\phi$ is u.h.c. and $Z \times E$ is compact, $\phi(Z \times E)$ is compact, and so $\bigcup_{n=0}^{\infty} G_{n}$ is compact in $X^{E}$. Let $H=\bigcup_{n=0}^{\infty} G_{n} \cdot f_{n}$. Thus, $h \in H$ iff for some $n$ there exists a $g \in G_{n}$ such that $g_{n} \cdot f_{n}=h$. 


\section{Lemma 3.1. H is compact.}

Proof of Lemma. It suffices to show that (a) $H$ is pointwise closed, and (b) $\Pi_{w}(H)=\{h(w): h \in H\}$ is compact in $X$.

(a) Let $\left\{h_{\alpha}, \alpha \in \Lambda\right\}$ be a net in $H$ with limit $h$. Then $h_{\alpha}=g_{\alpha} \cdot f_{\alpha}$, where $g_{\alpha} \in G_{n}$ for some $n$ and $f_{\alpha}=f_{n}$. Since $F$ is compact, the net $\left\{f_{\alpha}, \alpha \in A\right\}$ has a convergent subnet $\left\{f_{\beta}, \beta \in \Lambda^{\prime}\right\}$ with limit $f \in F$. Then there exists a $g: E \rightarrow X$ such that the diagram

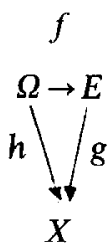

comments. Since $f=f_{n}$ for some $n$, it suffices to show that $g \in G_{n}$.

Consider the net $\left\{\left(x_{\beta}, z_{\beta}, f_{\beta}, h_{\beta}\right), \beta \in \Lambda^{\prime}\right\}$ where $\left(x_{\beta}, z_{\beta}\right)=\left(x_{n}, z_{n}\right)$ iff $h_{\beta}=g_{n} \cdot f_{n}$. Since $Y$ is compact there exists a subnet $\left\{\left(x_{\alpha}, z_{\alpha}, f_{\alpha}, h_{\alpha}\right), \alpha \in \Lambda^{\prime \prime}\right\}$ with limit $\left(x_{n}, y_{n}, f_{n}, h\right)$. For each $w,\left.\left(x_{\alpha}, y_{\alpha}, f_{\alpha}(w), h_{\alpha}(w)\right) \in \mathrm{Gr} \phi\right|_{z \times E}$. Since $\phi$ is u.h.c. and $Z \times E$ is compact, $\left.\operatorname{Gr} \phi\right|_{Z \times E}$ is compact. Hence, for all $w$, $\left.\left(x_{n}, z_{n}, f_{n}(w), h(w)\right) \in \operatorname{Gr} \phi\right|_{z \times E}$. Now $f_{n}(\Omega)$ and its complement are analytic sets (hence measurable). Define $\left.g\right|_{f_{n}}(\Omega)$ such that $g\left(f_{n}(w)\right)=h(w)$. Define $\left.g\right|_{f_{n}}(\Omega)_{c}$ to be an arbitrary measurable selection from $\left.\phi\left(x_{n}, z_{n}, \cdot\right)\right|_{f_{n}}(\Omega)_{c}$. Then $g$ so defined is a function in $G_{n}$, and $h=g \cdot f_{n}$, as was to be shown.

(b) Choose $w \cdot \Omega$. Then $\Pi_{w}(H)=\bigcup_{n=0}^{\infty}\left\{g_{n}\left(f_{n}(w)\right): g_{n} \in G_{n}\right\}=\phi(Z(w))$ which is compact since $Z(w)$ is compact and $\phi$ is u.h.c. This proves the lemma.

To complete the proof of the theorem, it suffices to show that $K(Z)$ is compact. But $K(Z)=\left\{\lambda \cdot h^{-1}: h^{-1} \in H\right\}$ which is compact since $H$ is compact. Q.E.D.

Proof of Theorem 3.2. Let $v \in M(E)$ bc atomlcss. It suffices to show that $v \cdot \phi^{-1}$ is convex. I need some intermediate propositions.

Lemma 3.2. Let $\left\{X_{1}, \ldots, X_{n}\right\}$ be a finite, measurable partition of $X$. Then the set $\left\{\mu\left(X_{1}\right), \ldots, \mu\left(X_{n}\right) ; \mu \in v \cdot \phi^{-1}\right\}$ is convex.

Proof of Lemma 3.2. Let $g_{1}, g_{2} \sim \phi$. Let $a_{i}=\left(v \cdot g_{i}^{-1}\left(X_{1}\right), \ldots, v \cdot g_{i}^{-1}\left(X_{n}\right)\right)$ for $i$ $=1,2$. We need to find for each $\alpha, 0 \leqq \alpha \leqq 1$, a measurable function $g \sim \phi$ such that $a=\left(v \cdot g^{-1}\left(X_{1}\right), \ldots, v \cdot g^{-1}\left(X_{n}\right)\right)=\alpha a_{1}+(1-\alpha) a_{2}$. 
Let $E^{i j}=g_{i}^{-1}\left(X_{i}\right)$ for $j=1,2, i=1, \ldots, n$. Let $E_{k l}=g_{1}^{-1}\left(X_{k}\right) \cap g_{2}^{-1}\left(X_{l}\right)$. Then $\left\{E_{k, l}\right\}_{k, l=1}^{n}$ is a measurable partition of $E$. Also, $\bigcup_{k} E_{k l}=E^{l, 2}, \bigcup_{l} E_{k l}=E^{k, 1}$, Finally, $v \cdot g_{j}^{-1}\left(X_{i}\right)=v\left(E^{i j}\right)$.

Since $v$ is atomless, there exists for all $\alpha, 0 \leqq \alpha \leqq 1$, sets $R_{k l} \subset E_{k l}$ such that $v\left(R_{k l}\right)=\alpha \nu\left(E_{k l}\right)$. Then the sets $\left\{R_{k, l}, E_{k, l} \backslash R_{k, l}\right\}_{k, l=1}^{n}$ form a measurable partition of $E$. Define $g$ as follows:

$$
\begin{aligned}
g(e) & =g_{1}(e), \quad e \in \bigcup_{k, l} R_{k, l}, \\
& =g_{2}(e), \quad e \in \bigcup_{k, l}\left(E_{k, l} \backslash R_{k, l}\right), \quad k, l=1, \ldots, n .
\end{aligned}
$$

Evidently $g$ is measurable, and $g \sim \phi$. Furthermore, $g^{-1}\left(X_{k}\right)$ $=\left(\bigcup_{l} R_{k l}\right) \cup\left(\bigcup_{l} E_{l k} \backslash R_{l k}\right)$. Thus

$$
\begin{aligned}
\nu \cdot g^{-1}\left(X_{k}\right) & =\sum_{l} v\left(R_{k l}\right)+\sum_{l} v\left(E_{l k} \backslash R_{l k}\right) \\
& =\alpha \sum_{l} \nu\left(E_{k l}\right)+\alpha(1-\alpha) \sum_{l} \nu\left(E_{l k}\right) \\
& =\alpha \nu\left(E^{k 1}\right)+(1-\alpha) \nu\left(E^{k 2}\right) \\
& =\alpha \nu \cdot g_{1}^{-1}\left(X_{k}\right)+(1-\alpha) \nu \cdot g_{2}^{-1}\left(X_{k}\right) .
\end{aligned}
$$

Since this holds for all $k$, the Proposition is proved. Q.E.D.

An immediate consequence of Lemma 3.2 is:

Lemma 3.3. Let $\left\{X_{1}, \ldots, X_{n}\right\}$ be a measurable partition of $X$, and let $f_{1}, \ldots, f_{k}$ be $k$ functions such that $f_{i}()=c_{i j}$ for $x \in X_{j}$. Then the set $\left\{\int f_{1} \mathrm{~d} \mu, \ldots, \int f_{k} \mathrm{~d} \mu\right.$; $\left.\mu \in v \cdot \phi^{-1}\right\}$ is convex.

Proof of Lemma 3.3. We have $\int f_{i} \mathrm{~d} \mu=\sum_{j} c_{i j} \mu\left(X_{j}\right)$. The result then follows from Lemma 3.2. Q.E.D.

Lemma 3.4. Let $h_{1}, \ldots, h_{k}$ be $k$ functions founded and continuous from $X$ into $R$. Then $\left\{\int_{1} \mathrm{~d} \mu, \ldots, \int h_{k} \mathrm{~d} \mu ; \mu \in v \cdot \phi^{-1}\right\}$ is convex.

Proof of Lemma 3.4. There exists a sequence of functions $\left\{\left(f_{1}^{n}, \ldots, f_{k}^{n}\right)\right\}_{n=1}^{\infty}$, each set of functions $\left(f_{1}^{n}, \ldots, f_{k}^{n}\right)$ constant on members of a partition $\left\{X_{1}^{n}, \ldots, X_{n}^{n}\right\}$ of $X$, and such that $f_{i}^{n} \rightarrow h_{i}$ uniformly. Let $A_{n}=\left\{f_{1}^{n} \mathrm{~d} \mu, \ldots, f_{k}^{n} \mathrm{~d} \mu\right.$; $\left.\mu \in v \cdot \phi^{-1}\right\}$. From Theorem 3.1 each $A_{n}$ is closed. Let $A=\lim _{k} A_{k}$. Thus $a \in A$ iff there is a sequence $\left\{\mu_{n}\right\} \subset v \cdot \phi^{-1}, a=\lim _{n}\left(\int f_{1}^{n} \mathrm{~d} \mu_{n}, \ldots, \int f_{k}^{n} \mathrm{~d} \mu_{n}\right)$. Since $v \cdot \phi^{-1}$ is compact, there is a subsequence $\left\{\mu_{m}\right\} \subset\left\{\mu_{n}\right\}, \mu_{m} \rightarrow \mu \in v \cdot \phi^{-1}$. For each $i,\left\|\int f_{i}^{m} \mathrm{~d} \mu_{m}-\int h_{i} \mathrm{~d} \mu\right\| \leqq \int\left\|f_{i}^{m}-h_{i}\right\| \mathrm{d} \mu_{m}+\left\|\int h_{i} \mathrm{~d} \mu_{m}-\int h_{i} \mathrm{~d} \mu\right\|$. Each term converges to 0 , and so $\lim _{m} \int f_{i}^{m} \mathrm{~d} \mu_{m}=\int h_{i} \mathrm{~d} \mu$. Thus $a \in A$ iff $a$ 
$=\left(\int h_{i} \mathrm{~d} \mu, \ldots, \int h_{k} \mathrm{~d} \mu\right)$ for some $\mu \in v \cdot \phi^{-1}$. Since each $A_{k}$ is closed and convex (Theorem 3.1 and Lemma 3.3), $A$ is convex. Q.E.D.

Let $\left\{h_{i}\right\}_{i=1}^{\infty}$ be a dense set of continuous functions from $X$ into the unit interval $I$. Let $R_{n}(\phi)=\left\{\left(\int h_{1} \mathrm{~d} \mu, \ldots, \int h_{n} \mathrm{~d} \mu\right) ; \mu \in v \cdot \phi^{-1}\right\} \times I \times I \times \ldots$. Then $R_{n}(\phi)$ is convex subset of the Hilbert cube. Let $R(\phi)=\left\{\left(\int h_{1} \mathrm{~d} \mu, \ldots\right)\right.$; $\left.\mu \in v \cdot \phi^{-1}\right\}$. Then $R(\phi)=\bigcap_{n} R_{n}(\phi)$, and so $R(\phi)$ is a convex subset of the Hilbert cube. Define the map $\lambda: M(X) \rightarrow \prod_{i=1}^{\infty} I$ by $\lambda(\mu)=\left(\int h_{1} \mathrm{~d} \mu, \ldots\right)$. This map is continuous, linear, and invertible. Thus $v \cdot \phi^{-1}=\lambda^{-1}(R(\phi))$, and so $v \cdot \phi^{-1}$ is convex. Q.E.D.

This concludes the proof of Theorem 3.2.

\section{Applications}

In this section I give an application of the techniques presented in sections 2 and 3 to stochastic equilibrium analysis.

In the usual temporary equilibrium framework [Grandmont (1977)] there is a compact subset $D \subset \boldsymbol{R}^{m}$ of endogenous variables and a compact subset $E$ $\subset \boldsymbol{R}^{n}$ of exogenous variables. The state space for the Markov equilibrium process is $X=E \times D$. The evolution of the stochastic equilibrium process is determined by an upper hemi-continuous correspondence $\phi: E \times X \rightarrow X$ and by a transition probability $\psi: E \times \sigma(E) \rightarrow[0,1]$. The correspondence $\phi$ is the equilibrium correspondence, and $\phi\left(x_{t-1}, e_{t}\right)$ is the set of all possible equilibrium states $x_{t}$ that can occur at time $t$ if the state at time $t-1$ was $x_{t-1}$ and exogenous random variable $e_{t}$ is realized at time $t$. The transition probability $\psi$ describes the Markovian evolution of the exogenous random variable.

The tradition approach has been to choose a measurable selection $f \sim \phi$, and to describe the evolution of equilibrium states by the transition probability

$$
K_{f}(x, \cdot)=\psi(x) \cdot f^{-1}(x, \cdot)
$$

The problem to be addressed is the existence of a stationary distribution of states. Does there exist a selection $f$ from $\phi$ such that $K_{f}$ admits an invariant distribution? This question was first addressed by Grandmont and Hildenbrand (1974), who showed that if $\psi$ has the Feller property and the selection $f$ is continuous, $K_{f}$ satisfies the Feller property, and thus admits an invariant distribution. However, the existence of a continuous selection tantamount to the uniqueness of equilibrium in each state - is not often satisfied. Theorems 3.1, 3.2 and 2.1 together imply that, under the additional assumption that for all $x \in X, \psi(x)$ is atomless, there always exists a selection $f \sim \phi$ such that $K_{f}$ admits an invariant probability. 
The results of the previous section can also be used to solve a problem considered by several authors: Blume (1977), Easley (1978), and Knieps (1977). The problem is the existence of weakly rational expectations. ${ }^{1}$

Recall that the state space is $X=E \times D$, where $D$ is the set of endogenous variables. The endogenous variables are of two types: those that are actions of individual agents, and those that are jointly determined by the actions of the agents (such as prices). Each agent is determining his optimal action by observing current jointly determined and some current exogenous variables, and by solving a dynamic programming problem. To solve this problem, agents need to have some expectations about those variables they observe. Let there be $n$ agents, and let $S_{i}$ be the set of variables that the $i$ th agent observes. We assume that agents do not have beliefs about the dynamic evolution of the $s_{i t}$, and so the expectations of the $i$ th agent can be represented by probability distributions $\mu_{i} \in M\left(S_{i}\right)$, the set of probability distributions on $S_{i}$. Thus the term 'weakly' in the title of the problem. However, it does seem plausible that in a stationary equilibrium, agents should know the true marginal distribution of $s_{i}$ from the invariant probability measure. Thus the word 'rational'. Our problem, then, is to find a stationary equilibrium where agents have the correct marginal distribution of the observed variables as their expectations.

This can be studied as a fixed point problem. Give $M\left(S_{i}\right)$ the topology of weak convergence. Then let $M=\prod_{i=1}^{n} M\left(S_{i}\right), v \in M$ is a vector $\left(v_{1}, \ldots, v_{n}\right)$ of probabilities on the $S_{i}$. We derive an equilibrium correspondence $\phi: X \times E$ $\times M \rightarrow X$, and, because the world is simple, $\phi$ is upper hemi-continuous. See Blume (1977), Easley (1978) or Knieps (1977) for details. If we assume that $\psi$ has atomless range, then $K(x, v)$ is compact, convex and non-empty valued, and upper hemi-continuous (Theorem 3.2).

Let $\rho: M(X) \rightarrow M$ be the map such that $\rho(\mu)$ is the vector of marginal distributions of $\mu$ on $S_{1}, \ldots, S^{n}$, respectively. The map $\rho$ is continuous, and so the correspondence $K(x, \rho(\mu)): X \times M(X) \rightarrow M(X)$ is upper hemi-continuous, etc. Now define $A: M(X) \times M(X) \rightarrow M(X)$ such that $\Lambda\left(\mu, \mu^{\prime}\right)$ $=\int K\left(X, \rho\left(\mu^{\prime}\right)\right) \mu(\mathrm{d} x)$. Then $A$ is upper hemi-continuous, convex and compact and non-empty valued. In particular, its restriction to the diagonal of $M(X)$ $\times M(X)$ enjoys these properties. By identifying $M(X)$ with the diagonal of $M(X) \times M(X)$ we have a correspondence $\tilde{A}: M(X) \rightarrow M(X)$ which satisfies all the conditions of Ky Fan's Fixed Point Theorem. Thus, there exists a $\mu \in M(X)$ such that $\mu \in \int K(x, \rho(\mu)) \mu(\mathrm{d} x)$. In other words, $\mu$ is a stationary equilibrium in which each agent knows the correct one-period distribution of his observable variables.

In earlier work, Blume (1977), Easley (1978) and Knieps (1977) all had to choose a continuous $f \sim \phi$. Here we have demonstrated the existence of a

\footnotetext{
'So named by Easley.
} 
selection $h \sim \phi$ such that the transition probability constructed from it admits a weakly rational expectations equilibrium. The cost of weakening the requirements on selections from $\phi$ is only the stronger requirement on $\psi$. In fact, the development of these techniques was motivated by a study by the author of rational expectations when agents are aware of the dynamic structure of the economy [Blume (1979)]. In this case, where transition probabilities on the $S_{i}$ must be considered, it is clear that non-trivial examples admitting continuous selections exist.

\section{References}

Blume, L., 1977, The stationarity of Markov processes of temporary equilibrium, IBER/CRMS working paper 252 (University of California, Berkeley, CA).

Blume, L., 1979, Consistent expectations, Unpublished paper (University of Michigan, Ann Arbor, MI).

Easley, D., 1978, The formation of expectations in an uncertain environment, Unpublished Ph.D. thesis (Northwestern University, Chicago, IL).

Grandmont, J.M., 1977, Temporary general equilibrium, Econometrica 45, no. 3, 535-572.

Grandmont, J.M. and W. Hildenbrand, 1974, Stochastic processes of temporary equilibrium, Journal of Mathematical Economics 1, no. 3, 247-277.

Hildenbrand, W., 1974, Core and equilibria of a large economy (Princeton, NJ).

Knieps, G., 1977, Unpublished Ph.D. thesis (University of Bonn, Bonn). 